\title{
A New NRI Metamaterial-based Ultra Wideband Stop Band Filter
}

\author{
Sikder Sunbeam Islam*, Mohammad Rashed Iqbal Faruque, and Mohammad \\ Tariqul Islam \\ Space Science Centre, University Kebangsaan Malaysia, \\ Bangi-43600, Selangor, Malaysia \\ sikder_islam@yahoo.co.uk, rashedgen@yahoo.com, titareq@yahoo.com
}

\begin{abstract}
In this paper, a metamaterial-based ultra wideband stop-band filter is presented. Initially, a pi-shaped metamaterial was designed. The metamaterial shows wide band negative refractive index and near zero refractive index property. The metamaterial was then utilized in designing a filter that performs stop-band operation over an ultra wide band region. The smaller size ultra wideband application has persuaded the design a potential one.
\end{abstract}

Keywords: Metamaterials; Filter; Ultra-wideband;

\section{Introduction}

Artificially constructed metamateraial have become a promising field for the researcher. Metamaterial is playing a vital role in almost all the electromagnetic field due to its exceptional electromagnetic properties. Materials those are found in nature rarely show negative permittivity or negative permeability but not simultaneously. Moreover, natural material with refractive index of a negative value is not available. Natural material having simultaneous negative permittivity and permeability is unavailable well. However, artificially developed a metamaterial have overcome all these complexities and may contain all these extraordinary characteristics simultaneously. For this reason, now almost in every field of electromagnetism metamaterial is being used like, in antenna design, cloak design, sensing application, filter design etc. [1-5].

Metmaterial can be utilized in designing a wideband filter. In the field of filter design, very few works are found with metamaterial in stop-band applications. For example, a metamaterial-based cut-band filter was proposed in [6] for C-band applications. They utilized a different array of same metamaterial for increasing the resonance, but all in C-band of microwave spectra. They also increased bandwidth by increasing the metamaterial array. A metamaterial-based stop band filter was reported

* Please note that the LNICST Editorial assumes that all authors have used the western naming convention, with given names preceding surnames. This determines the structure of the names in the running heads and the author index. 
in [7], but the centre frequency of their filter operates in the S-band region only. They utilized four open loop resonators in the upper and lower side of the feed line to get the poles of the pass band of the filter. A metamaterial-based stop-band filter was proposed in [8], where two electrical metamaterial unit cells were utilized in the filter design in the form of fractal resonator to create the stop band in the L-band only. However, they did not provide any metamaterial characteristics.

In this study, a new pi-shaped metamaterial-based stop-band filter is presented. Initially, a pi-shaped metamaterial unit cell was designed. Then the unit cell was employed for the design of a stop-band filter. Finite integration technique based simulation software CST was used for the design and S-parameters calculation of the unit cell and filter.

\section{Design of the Unit Cell}

In the Fig.1, the structure of the proposed metamaterial unit cell is being displayed. This structure is based on pi-shape design. " $\pi$ " is term, is usually used in mathematical calculation specially in the geometrical aspect. The proposed pi-shaped structure was developed on FR-4 substrate material having relative permittivity $\varepsilon=4.2$ and the thickness of $1.6 \mathrm{~mm}$. Loss tangent of the material was 0.002 . The dimension of the substrate was maintained $10 \mathrm{~mm}$. The pi-shape unit cell used here contains two metal L-shaped arms arranged in an inverted pattern to each other. Tiny gap was maintained between the arms at two ends. The dimension of the unit cell is shown in Table-1.

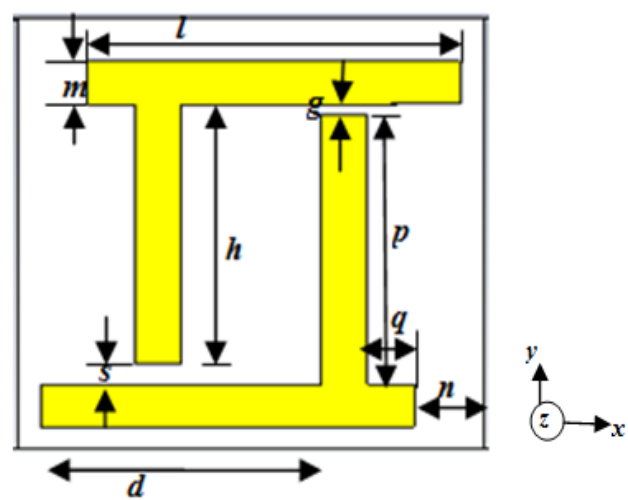

Fig. 1. The proposed metamaterial unit cell 
Table 1. Paramaters of the Unit cell

\begin{tabular}{cc}
\hline Unit Cell Parameters & Value $(\mathrm{mm})$ \\
\hline $\mathrm{d}$ & 6 \\
$\mathrm{~g}$ & 0.33 \\
$\mathrm{~h}$ & 6 \\
$\mathrm{l}$ & 8 \\
$\mathrm{~m}$ & 1 \\
$\mathrm{n}$ & 1.5 \\
$\mathrm{p}$ & 6.27 \\
$\mathrm{q}$ & 1 \\
$\mathrm{~s}$ & 0.5 \\
\hline
\end{tabular}

The size of the unit cell was chosen to have more than three times shorter than the wavelength. The length of the arms creates magnetic resonance and the gap between the arms produce electrical resonance.

\section{Methodology}

To excite a structure properly, the incident electric field should be polarized along the $x$-axis and the magnetic field along the $y$-axis. The electromagnetic wave should be propagated along the $z$-axis. Therefore, simulation of the unit cell was done using CST Microwave Studio and transverse electromagnetic wave was propagated through the z-axis using two waveguide ports. For the measurement reason, a metamaterial prototype consisting $23 \times 12$ unit cells was prepared. The fabricated prototype for measurement is shown in Fig.2. Two horn antennas were used as a transmitter and receiver of electromagnetic waves and those were connected to a vector network analyzer Agilent E8363D. The fabricated prototype of pi-shaped metamaterial was placed between the antennas to extract the experimental S-parameters.

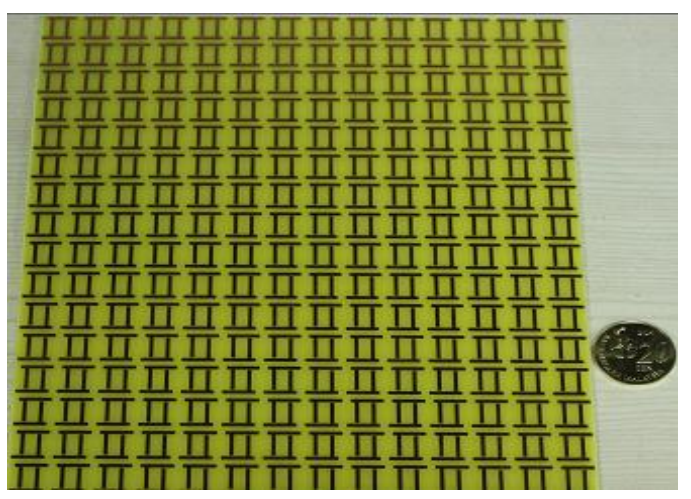

Fig. 2.Fabricated pi-shaped metamaterial prototype for measurement 


\section{Result and Discussion}

The S-parameter results of the simulation are shown in Fig.3. Two transmittances in $8.31 \mathrm{GHz}$ and 12.81 $\mathrm{GHz}$ in the microwave region are seen from the Fig.3. The measured results of the S-parameters are provided in the same figure as well for the comparison. The experimental results show almost good conformity with the simulation results.

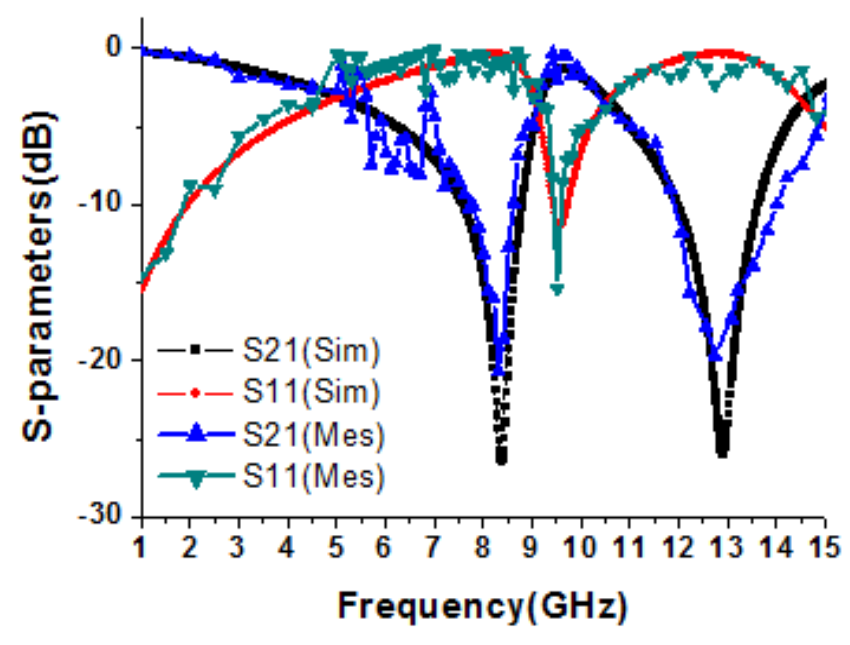

Fig. 2. S-Parameter magnitude of the pi-shaped unit cell in $\mathrm{dB}$

The Fig.4 depicts the map of the surface current distribution in the proposed pi-shaped meta-atom at $12 \mathrm{GHz}$. From that figure, it is seen that the direction of current along the two sides of the unit cell is opposite that yields sharp transmittance for the unit cell.

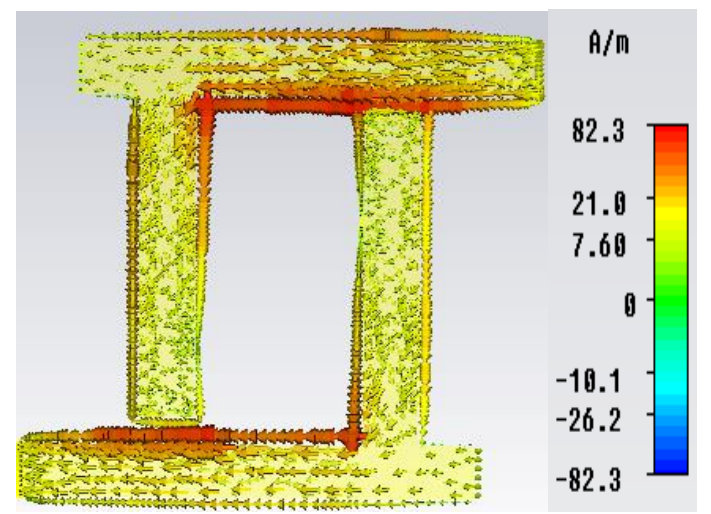

Fig. 3. Surface current distribution in pi-shaped atom 
The numerical results of the S-parameters were utilized in a slandered retrieval technique- NicolsonRoss-Weir (NRW) algorithm [9] to evaluate the real and imaginary value of effective permeability and permittivity. Direct calculation of refractive index was done using the direct refractive index (DRI) algorithm mentioned in [10] for perfection.

In the Fig. 5a and 5b, the permittivity and permeability curves against frequency are shown. The real permittivity curve shows a negative region from $4.58 \mathrm{GHz}$ to $8.48 \mathrm{GHz}, 9.38 \mathrm{GHz}$ to $11.33 \mathrm{GHz}$ and 14.29 $\mathrm{GHz}$ to $15 \mathrm{GHz}$ frequency. Similarly, the real mu-negative curve is seen in the Fig.5b from $8.85 \mathrm{GHz}$ to 15 $\mathrm{GHz}$ frequencies. Therefore, the common negative area of the both permittivity and permeability curve may produce negative refractive index (NRI) zone in the range. The imaginary curves of both permittivity and permeability curve refers to the electric and magnetic loss tangent of the material.

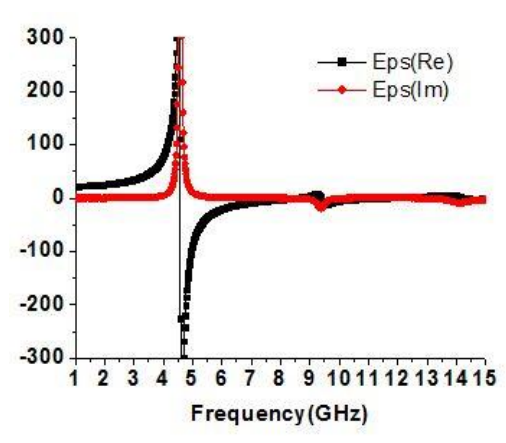

(a)

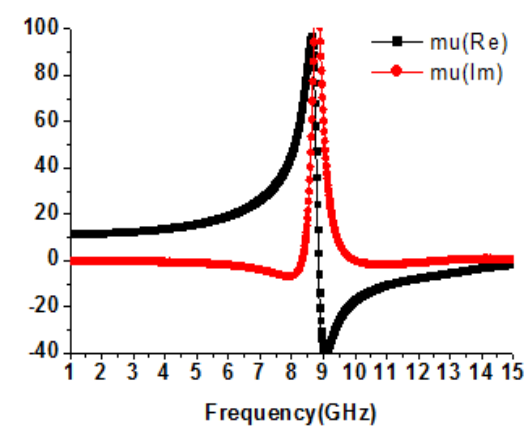

(b)

Fig. 4. (a) Effective permittivity, (b) effective permeability of the pi-shape metamaterial unit cell.

The Fig. 6 shows the refractive index curve for the pi-shaped atom of the metamaterial. Negative refractive index (NRI) region is visible in the frequency range of $9.38 \mathrm{GHz}$ to $11.59 \mathrm{GHz}$ and $12.57 \mathrm{GHz}$ to $15 \mathrm{GHz}$. This range covers the $\mathrm{X}$-and Ku-band of microwave regime. Usually, in this NRI region, the energy reverses to the phase of the waves. The Imaginary part of the refractive index curve is found positive over the whole frequency in the range. Moreover, near zero region of the refractive index is also visible in the frequency range of $5 \mathrm{GHz}$ to $8.5 \mathrm{GHz}$ region. 


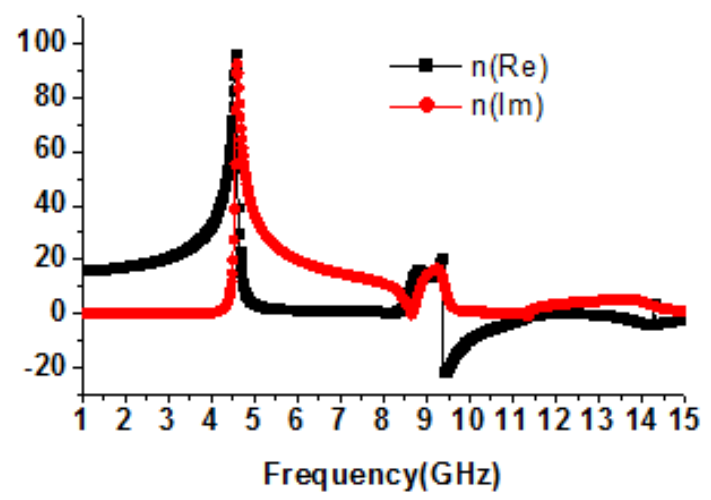

Fig. 5. Refractive index (n) curve for the pi-shaped atom

\section{Metamaterial-based Filter Design and Operation}

In a further step, a filter was constructed using the metamaterial unit cell for stop-band operation. The $20 \mathrm{~mm} \times 10 \mathrm{~mm}$ filter was designed on the same substrate material. However, in the filter design the copper arms of the unit cell structure were extended up to ending border on two opposite sides.

Two waveguide ports were connected to the ends of the arms of the filter. The two ports are acting as transmitter and receiver of the filter. The design and simulation arrangement is being displayed in the Fig.7. The same electromagnetic wave was propagated through the filter and S-parameters were calculated.

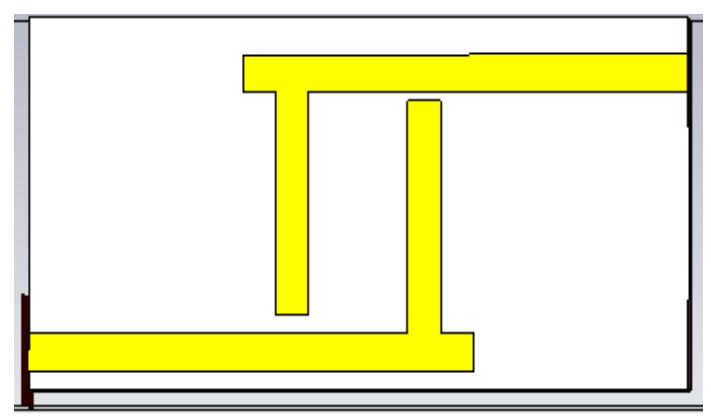

Fig. 6. Simulation setup of the metamaterial-based filter

The current distribution for the filter operation at $12 \mathrm{GHz}$ is shown in the Fig.8. It is seen from the Fig. 8 that the current following opposite dirrection that results wide 
cut-band region of the filter. However, the current distribution follows the same of metamaterial unit cells, which reflects the direct contribution of the metamaterial for the filter operation.

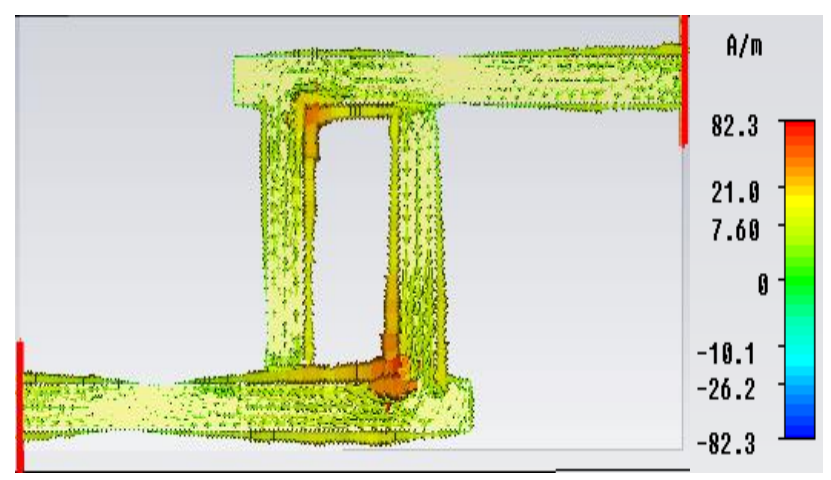

Fig. 7. Current distribution for the filter operation

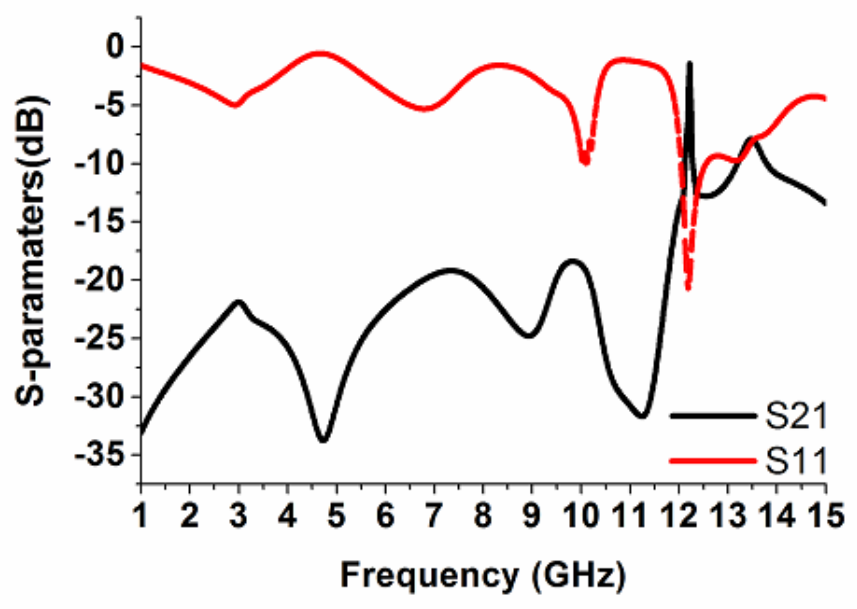

Fig. 8. S-paramaters for the filter operation

The Fig.9 displays the numerical results of S-parameters for the filer operation. The stop band region has started from the frequency of $1 \mathrm{GHz}$ to $12.04 \mathrm{GHz}$. The rejection level was found greater than $20 \mathrm{~dB}$ from $1 \mathrm{GHz}$ to $10 \mathrm{GHz}$. The return loss was found below $-10 \mathrm{~dB}$ from the frequency of $12.07 \mathrm{GHz}$ to $12.55 \mathrm{GHz}$. A small pass band is visible from the frequency of $12.15 \mathrm{GHz}$ to $12.28 \mathrm{GHz}$. 


\section{Conclusion}

In this paper, a new negative refractive index property (NRI) based-metamaterial is proposed. The characteristics of permittivity, permeability of the metamaterial supports the refractive index property of the material for the wideband region X-and Ku-band in the microwave span. Experimental results were also provided. Later on, an ultra stop band filter was designed using the metamaterial that is applicable for ultra wideband applications. The filter operating with rejection over $12 \mathrm{GHz}$ areas in conjunction with small pass band region. Therefore, the metamaterial can be utilized for filter applications beside other metamaterial applications.

\section{References}

1. Veselago, V. G.: The electrodynamics of substances with simultaneously negative values of $\varepsilon$ and $\mu$, Sov. Phys. Usp., 10, 509-514 (1968)

2. Islam, S. S., Faruque, M. R. I., Islam, M. T.: A Near Zero Refractive Index Metamaterial for Electromagnetic Invisibility Cloaking Operation; Materials, 8, 4790-4804 ( 2015)

3. Chen, H., Ran, L., Huangfu, J., Zhang, X., Chen, K.: Left-handed materials composed of only Sshaped resonators, Phys. Rev. E, 70, 057605:1-4(2004)

4. Islam, S. S., Faruque, M. R. I., Islam, M. T.: An Object-Independent ENZ Metamaterial-Based Wideband Electromagnetic Cloak, Sci. Rep. 6, 33624:1-10 (2016)

5. Chen , T. , Li, S., Sun, H.: Metamaterials application in sensing, Sensors, 12, 27422765 (2012)

6. Carver, J., Reignault, V.: Engineering of the metamaterial-based cut-band filter; Appl. Phys. A, 117,513-516 (2014)

7. Gorur, A.K., Karpuz, C., Ozek, A., Emur, M.: Metamaterial based dual-band bandpass filter design for WLAN/WiMAX applications; Microw. Opt. Technol. Lett. 56, 2211-2214 (2014)

8. Palandoken, M., Sondas, A.: Compact metamaterial-bansed stop band filetr; Microwave J.; 57, 76-84 (2014)

9. R. W. Ziolkowski; Design, Fabrication, and Testing of Double Negative Metamaterials; IEEE T. Antenn. Propag. 51, 1516-1529( 2003)

10. Islam, S. S., Faruque, M. R. I., Islam, M. T.: 'A New Direct Retrieval Method of Refractive Index for Metamaterials', Curr. Sci., 109,337-342 (2015) 